\title{
CONSTRUCTION OF REGIONAL WEIGHTED MEAN TEMPERATURE MODEL BASED ON OPTIMIZATION BP NEURAL NETWORK
}

\author{
Zixin Chen $^{1,2}$, Lilong Liu ${ }^{1,2 *}$, Liangke Huang ${ }^{1,2,3}$, Qingtong Wan ${ }^{1,2}$, Xuqi Mo ${ }^{1,2}$ \\ College of Geomatics and Geoinformation, Guilin University of Technology, Guilin 541004, China \\ Guangxi Key Laboratory of Spatial Information and Geomatics, Guilin, 541004, China \\ GNSS Research Center, WuHan University, WuHan, 430079, China
}

KEY WORDS: Weighted Mean Temperature; BP Neural Network; Precipitable Water Vapor; China Region; Radiosonde

\begin{abstract}
:
The tropospheric weighted mean temperature $(\mathrm{Tm})$ is one of the key characteristic parameters in the troposphere, which plays an important role in the conversion of Zenith Wet Delay (ZWD) to atmospheric Precipitable Water Vapor (PWV). The precision of Global Navigation Satellite System (GNSS) inversion of PWV can be significantly improved with the accurate calculation of Tm. Due to the strong nonlinear mapping ability of Back Propagation (BP) neural network, the algorithm can be used to excavate the law with massive data. In term of the nonlinear and non-stationary characteristics of GNSS precipitable water vapor, in this paper, we proposes a forecast method of GNSS precipitable water vapor based on BP neural network, which can modelling the weighted mean temperature of troposphere. The traditional BP neural network has some shortcomings, such as large amount of calculation, long training time and easy to appear "over-fitting" phenomenon and so on. In order to optimize the deficiency and numerical simulation, the three characteristic values include water vapor pressure, surface pressure and surface temperature provided are selected as input parameters, named as BP_Tm. The optimal initialization parameters of the model were obtained from the 2016 radiosonde data of 89 radiosonde stations in China, and the modeling and accuracy verification were conducted with the 2017 radiosonde data, and the accuracy of the new model was compared with the common regional Tm model. The results show the BP_Tm model has good simulation accuracy, the average deviation is $-0.186 \mathrm{~K}$, and the root mean square error is $3.144 \mathrm{~K}$. When simulating the weighted mean temperature of a single station, the accuracy of the four models to simulate Tm is compared and analyzed, which the BP Tm model can obtain good accuracy and reflect better stability and reliability.
\end{abstract}

\section{INTRODUCTION}

Water vapor, an important components of the atmospheric system which directly related to rainfall. It is an extremely rapidly changing greenhouse gas, mainly distributed at the bottom of the troposphere, together with carbon dioxide as an important indicator of the global warming effect. The distribution and variation of water vapor have a great impact on

\footnotetext{
* Corresponding author
}

climate change. The development, formation and evolution of many natural disasters are closely related to atmospheric water vapor content. How to accurately know the temporal and spatial distribution characteristics of atmospheric water vapor and its changing laws is one of the important issues that need further research and analysis in the field of meteorology. With the rapid development of GNSS, the use of GNSS technology for water vapor detection has advantages of high spatial and temporal resolution, all-weather condictions, near real-time, continuity, high precision and become a new means of detection, When GNSS signal passes through the troposphere, 
the propagation path will be changed due to refraction, and the propagation speed will be relatively slow, eventually leading to the signal receiving time delay, which is commonly known as zenith total delay (ZTD). The zenith total delay consists of zenith hydrostatic delay (ZHD) and zenith wet delay (ZWD). The zenith hydrostatic delay (ZHD) can be accurately obtained using empirical models. ZWD can be obtained by separating ZHD from ZTD. ZWD has a linear relationship with PWV and can be represented by a conversion parameter $\Pi$. Since the atmospheric weighted mean temperature is the only variable for calculating $\Pi$, accurate acquisition of Tm can obtain accurate precipitation, which plays an important role in the GNSS inversion of atmospheric precipitation. At present, the commonly used Tm research methods mainly include statistical analysis methods based on radiosonde data and numerical simulation methods based on atmospheric reanalysis data. The research data can be obtained from numerical weather prediction (NWP) products, radiosonde data and meteorological reanalysis data. The distribution of radiosensor stations in the world is uneven, and there is almost no reliable data on the ocean, and its spatial resolution is difficult to meet the needs of GNSS stations. Therefore, NWP products or reanalysis data have become an important means for GNSS stations to obtain high-precision Tm. However, NWP products or reanalysis data have low time resolution problems, which cannot meet near-real-time meteorological observation requirements. Therefore, scholars at home and abroad have done many studies on $\mathrm{Tm}$ and established global and regional Tm models. Bevis et al $(1992,1994)$ proposed the principle and method of estimating the atmospheric water vapor content by using ground-based GPS technology, and constructed a linear function model of Tm-Ts based on the linear relationship between Tm and Ts. However, later scholars found that the relationship between $\mathrm{Tm}$ and Ts fluctuated with the change of station elevation and latitude, and the model had high accuracy only in a special area $\left(27^{\circ} \mathrm{N} \sim 65^{\circ} \mathrm{N}\right)$. Many scholars established a weighted mean temperature model for the region. Yao et al (2015) established a nonlinear functional relationship expression between $\mathrm{Tm}$ and Ts through the combination of derivation and mathematical statistics. Yao et al (2014) constructed a GTm-III model based on spherical harmonics using GGOS Atmosphere grid data. Chen et al (2014) uses the NCEP reanalysis data to established the global Tm model GWMT N based on the GWMT model. The above model requires a large amount of external grid data and NCEP reanalysis data, which requires high hardware and limits the ability to acquire $\mathrm{Tm}$ to a certain extent. Huang et al (2015) used the GGOS Atmosphere grid data to build a GGTm model based on the sliding window algorithm, which takes into account latitude and altitude changes with good accuracy.

In the field of artificial intelligence, some scholars have established an artificial neural network (ANN) by biologically mimicking the function of the brain structure and its processing information. In 1986, Rumelhart and other scholars independently proposed a BP neural network algorithm for multi-layer networks, which includes the input layer, hidden layer, and output layer topology. The weights and thresholds are modified by means of error back propagation to obtain a network model with the smallest sum of squared errors. BP neural network is a nonlinear mathematical model with adaptive, self-organizing and self-learning capabilities which has been widely used in many research fields such as pattern recognition, automatic control, signal processing, assisted decision making and artificial intelligence. In view of the non-linear and non-stationary characteristics of the available precipitation of the GNSS, a method for predicting the precipitation of the GNSS with the BP neural network is proposed in this paper. Based on the radiosonde data of 89 radiosonde stations in China in 2017, the weighted mean temperature is modeled, and the Tm model suitable for China is established, which is compared with other models to test the practicability and reliability of the model.

\section{METHODS FOR CALCULATING TM}

High-precision ZTD can be obtained by using GNSS technology and ZHD can be obtained by using an empirical model. ZWD can be obtained by separating ZHD from ZTD. The PWV can be multiplied by a ZWD with a conversion parameter $\Pi$ :

$$
\mathrm{PWV}=\Pi \cdot \mathrm{ZWD}
$$

$$
\Pi=\frac{10^{6}}{\rho_{w} R_{v}\left(\frac{k_{3}}{T_{m}}+k_{2}^{\prime}\right)}
$$

where $\mathrm{T}_{\mathrm{m}}$ is the weighted mean temperature of the troposphere; $r_{w}$ is the density of liquid water; $R_{v}$ is the gas parameter of water vapor; $k \notin, k_{3}$ is the atmospheric physical constant.

Davis J L et al (1985) gave the definition of Tm and the method for estimating $T_{m}$, which can be accurately calculated by the integral value of vapor pressure e and absolute temperature $T$ over the measuring station along the zenith direction:

$$
T_{m}=\frac{\int\left(\frac{e}{T}\right) d z}{\int\left(\frac{e}{T^{2}}\right) d z}
$$

where $\mathrm{e}$ is the vapor pressure, $\mathrm{T}$ is the absolute temperature, $\mathrm{z}$ is the vertical height above the station. 
According to the research results of many scholars at home and abroad, in the GNSS inversion of atmospheric water vapor content PWV, the calculation methods of $\mathrm{T}_{\mathrm{m}}$ are mainly as follows:1) constant value;2) approximate integration method;3) radiosonde data numerical integration;4) Bevis regression formula.

\section{CONSTRUCTION OF T MODEL BASED ON OPTIMIZED BP NEURAL NETWORK}

\subsection{Introduction of the BP neural network}

Artificial neural network is an adaptive nonlinear dynamic system composed of a large number of neurons through extremely rich and perfect connections. Mathematical methods are used to simulate biological neural networks from structure, implementation mechanism and function, thus establishing various network models. BP neural network is the most widely used algorithm, which has strong adaptability, distributed storage memory, parallelism and nonlinear mapping ability. When the $T_{m}$ model is established, the input is the surface pressure $\mathrm{P}$, the surface temperature $\mathrm{T}_{\mathrm{s}}$ and the water vapor pressure e of the modeling station, and the output is the $T_{m}$. The error is adjusted by the least square method using the gradient descent criterion. Figure 1 is a topological diagram of a neural network.

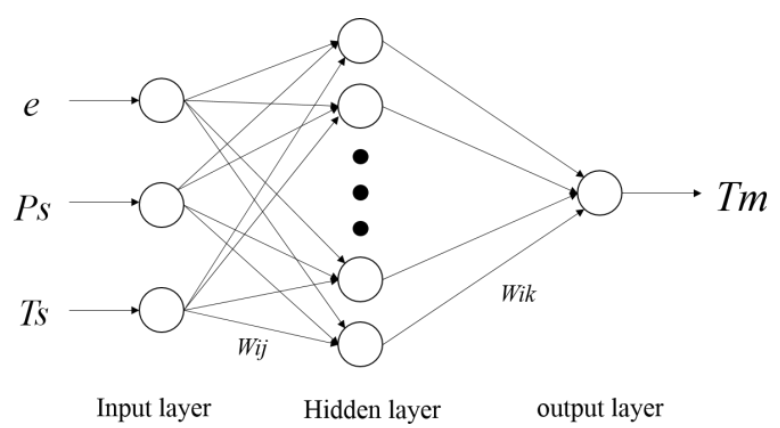

Figure 1. BP neural network topology diagram

In this paper the transfer function chosen is a nonlinear transformation function - Sigmoid function (also known as $\mathrm{S}$ function):

$$
f(x)=\frac{1}{\left(1+e^{-x}\right)}
$$

The $\mathrm{S}$ function can be used to map the data in $[0,1]$, so that the data is not easy to diverge during the transfer. The calculation formula of the input layer of each layer of BP neural network is as follows:

$$
I_{j}=\stackrel{\circ}{\text { 号 }}_{i=1}^{n} w_{i j} \times x_{i}+q_{j}
$$

where $\mathrm{n}$ is the number of network layers, $\mathrm{w}_{\mathrm{ij}}$ and $\theta_{\mathrm{j}}$ are the weights and thresholds of the nth layer, respectively, and $x_{i}$ is the input data, e, $\mathrm{p}_{\mathrm{s}}$, and $\mathrm{T}_{\mathrm{s}}$ in Figure 1. The output layer formula is:

$$
O_{j}=f\left(I_{j}\right)=\frac{1}{\left(1+e^{-I_{j}}\right)}
$$

where $\mathrm{O}_{\mathrm{j}}$ is the calculated $\mathrm{Tm}$. The weight calculation formula of $\mathrm{BP}$ neural network is

$$
w_{i j}(n+1)=w_{i j}(n)+h H_{j}(n) e_{k}(n)
$$

where $\eta$ is the learning rate.

At present, it is very difficult to accurately find the number of optimal hidden layer nodes. Generally, the range of the number of hidden layer nodes is obtained by the following empirical formula.

$$
\begin{gathered}
n_{1}=n+\sqrt{i+o} \\
n_{1}=a+(i+o)^{1 / 2} \\
n_{1}=2 i+1
\end{gathered}
$$

where $i$ is the number of input layer nodes, o is the number of output layer nodes, $\alpha$ is a constant between $[1,10]$, and $\mathrm{n} 1$ is the number of hidden layer nodes. The number of hidden layer nodes is between 3 and 12 by empirical formula.

\subsection{Introduction of the BP neural network}

The selection of hidden layer nodes, the adjustment of network parameters and the determination of initialization parameters in BP neural network are complex problems. In general, the number of hidden layer nodes can only be obtained by numerical simulation or empirical formula, too little will lead to 
network non-convergence or local minimum, too much will lead to network learning time is too long or simulation results are unstable. The setting of network parameters will affect the speed of network training and learning. In order to ensure the accuracy and efficiency of the model, the BP neural network optimized in this paper adopts the Levenberg-Marquardt optimization algorithm of nonlinear least squares method.
General BP neural network can adopt random initialization parameter, this will increase the network number of iterations, the error of slow convergence speed, reducing the speed of network learning, easy to fall in local best network, the model is difficult to reach the state of the global optimal and may occur in the process of calculating outliers, poor stability of the model. In order to solve the above problems, this paper uses historical observation data to perform multiple numerical simulations to obtain better model initialization parameters. The flow chart is shown in Figure 2.

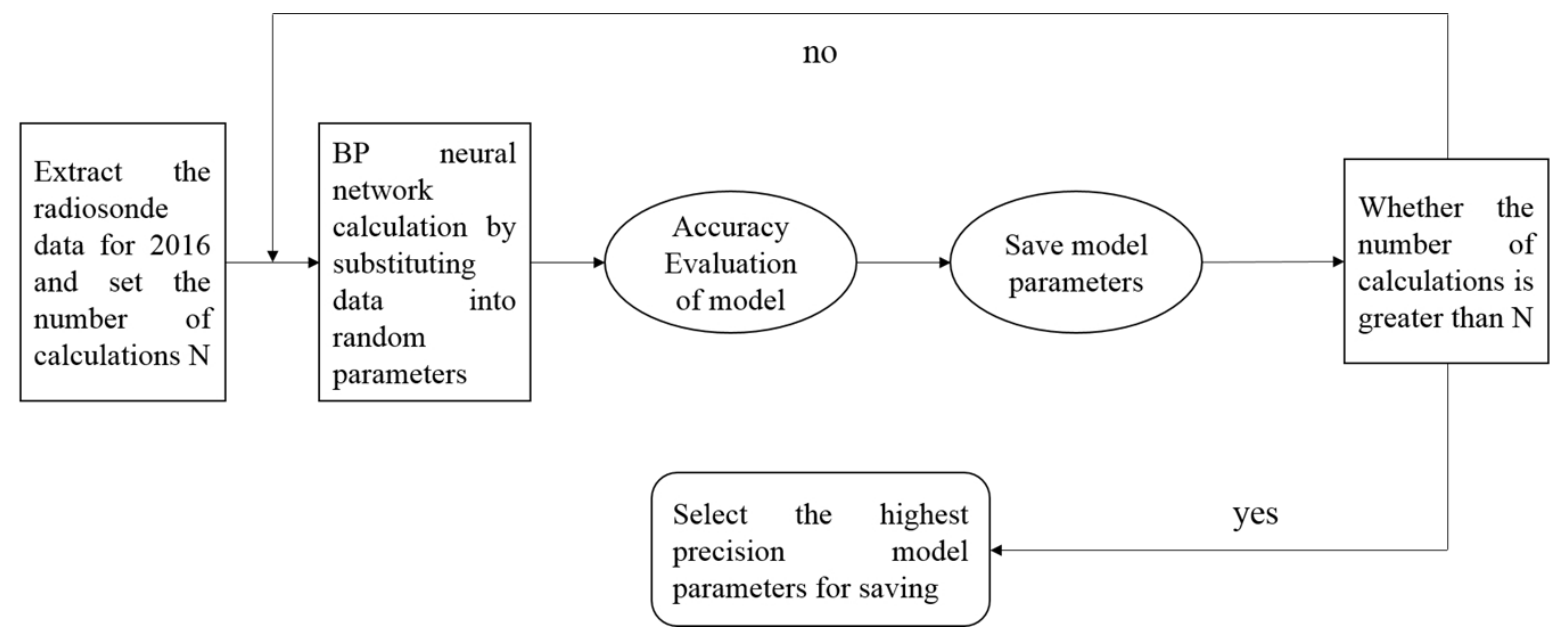

Figure 2. Optimized BP neural network initialization parameter flow chart

\section{VALIDATION OF BP_TM}

\subsection{Data Sources}

This paper chooses the data of 89 radiosonde stations in China from 2016 to 2017. The radiosonde data includes the height of the vertical direction of the atmosphere, temperature, atmospheric pressure, dew point, wind direction and wind speed at 00:00:00 and 12:00:00 (UTC). Using the radiosonde data of 2016 as historical data, the initial model parameters are obtained through numerical simulation, and the radiosonde data of 2017 is choosed to establish the model and evaluate the accuracy. The model was established by using the surface pressure $\mathrm{P}$, surface temperature Ts and water vapor pressure $\mathrm{e}$ of 76 stations. The surface pressure $\mathrm{P}$, surface temperature Ts and water vapor pressure e of 13 stations were used to evaluate the accuracy of the model. The distribution map of the radiosonde station is shown in Figure 3, which red part is the training station and the blue part is the inspection station. China has a vast territory, complex and diverse landforms, undulating terrain, and high height differences between sounding stations which normal height of each station ranges from 2 meters to 4,508 meters. The normal high distribution of the station is shown in Figure 4.

\subsection{Evaluation of Accuracy}

The weighted mean temperature calculated by the sounding integration method is taken as the true value. The accuracy of the Tm obtained by the BP model, Bevis model, Wang Xiaoying model and the nonlinear function model established by Yao Yibin are evaluated, respectively. The ground temperature Ts is measured by the sounding station. For convenience of description, the Bevis model is simply referred to as the B_Tm model, the Wang Xiaoying model is referred to as the W_Tm model, and the Yao Yibin model is referred to as the Y_Tm model. In this study, the bias and RMS error are regarded as criteria to assess the accuracy.

The linear relationship diagram and residual distribution map of the BP_Tm model simulation value and the sounding data integral value are shown in Figure 5. From Fig. 3(a), one can see that the model simulation value and the Tm value calculated by the radiosonde integration method show a good consistency, and there is no large difference. From Fig. 3(b), the residual of the BP_Tm model is mostly below $5 \mathrm{~K}$, and basically obeys the normal distribution expected to be zero. To verify the accuracy of the BP_Tm model, the bias and RMS of the four models are compared. The statistical results of different models are shown in Table 1 and Figure 6. 


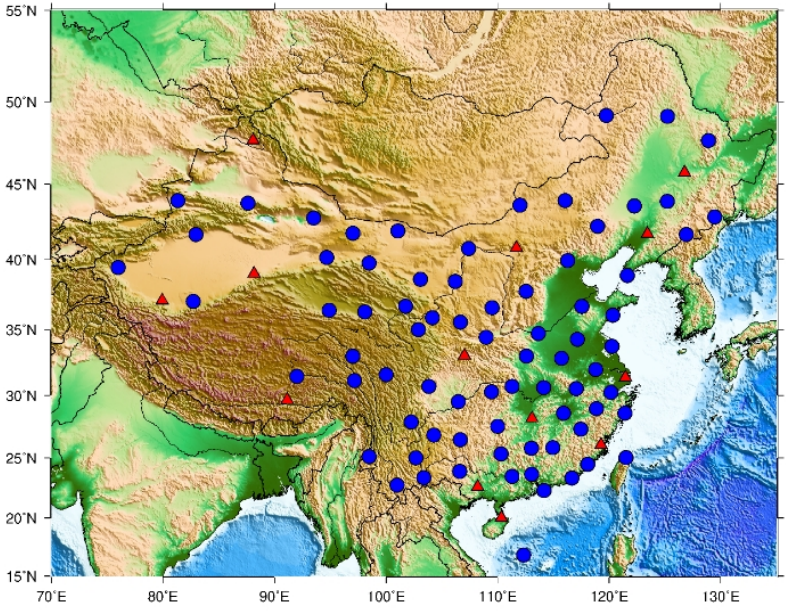

Figure 3. Distribution of selected 89 radiosonde stations in China
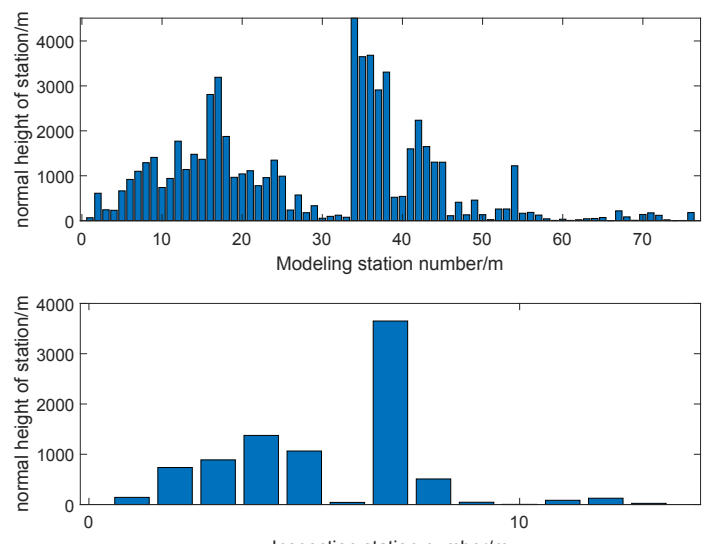

Figure 4. Normal height of training station and inspection station

From Table 1, one can see that the BIAS of the BP_Tm model is better than the other three models. The BIAS of the BP_Tm model is $-0.186 \mathrm{~K}$, the BIAS of the B Tm model is $0.668 \mathrm{~K}$, the BIAS of the W Tm model is $1.785 \mathrm{~K}$, and the BIAS of the Y_Tm model is $-0.224 \mathrm{~K}$. It shows that the BP_Tm model achieves good results in eliminating systematic errors. The Y_Tm model is better than the B_Tm model, and the W_Tm model has large systematic errors. The RMS of the BP_Tm model is $3.144 \mathrm{~K}$, which is $31 \%, 34 \%$, and $22 \%$ higher than the B_Tm model, W_Tm model, and Y_Tm model, respectively. The Y_Tm model is derived based on the assumption of the linear decreasing relationship of temperature with height, and the model fitting curve fluctuates with the surface temperature Ts. The B_Tm model is to establish the linear function relation of Tm-Ts after studying and analyzing the data of sounding stations in North America, and there is a certain deviation due to the difference of region. The Figure 6 show that the $\mathrm{W}_{-} \mathrm{Tm}$ model and the B_Tm model and the Y_Tm model have large RMS values in western China and northern China, which are mainly caused by latitude and highly undulating terrain. The BP_Tm model has a small difference in RMS values in southern China, and the RMS value in northern China is slightly larger. Overall, the BP_Tm model has good accuracy in China.

In order to verify the improvement effect of the BP_Tm model except for the sample station in China, the data of one station was excluded from the data of 76 sample test stations, and the integrity and non-specificity of the station data were guaranteed. Finally, the station was selected (111.3, 23.48, 120). After the optimization of BP neural network modeling, using the data of 215-245 days of annual product day in Wuzhou station in 2017, the simulation calculation and accuracy comparison of Tm were conducted by BP_Tm model and other three models. Figure 7 and Figure 8 respectively show the change in the simulated and true values of each model in Wuzhou station in August 2017.
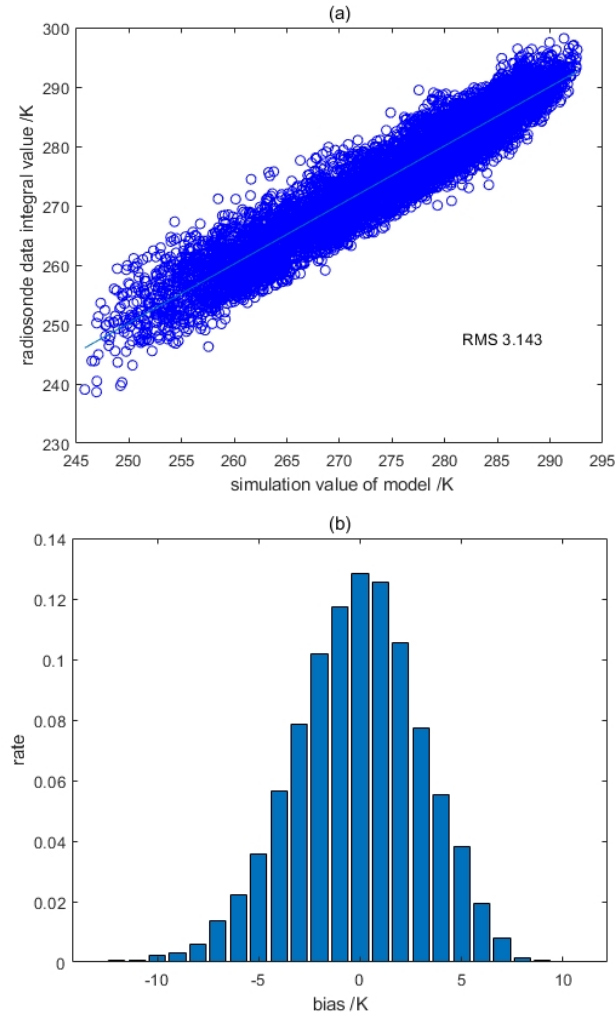

Figure 5. Relationship between model simulation value and sounding integral value and residual distribution map

\begin{tabular}{ccc}
\hline Tm & BIAS (K) & RMS (K) \\
\hline B_Tm & 0.668 & 4.567
\end{tabular}




$\begin{array}{lll}\text { W_Tm } & 1.785 & 4.810 \\ \text { Y_Tm } & -0.224 & 4.459 \\ \text { BP_Tm } & -0.186 & 3.144\end{array}$

Table 1. Accuracy statistical analysis of different models

As can be seen from Figure 7 and Figure 8, the Tm simulation value of the BP_Tm model has non-linear fluctuations similar to the actual situation, which is basically consistent with the curve trend of the true value, without any major deviation. The curve trend of simulated values of the W_Tm model, the B_Tm model and the Y_Tm model are basically the same, but the simulated values of the three have a large difference compared with the true value. Both B_Tm model and W_Tm model are linear function models of Tm_Ts while Y_Tm model is a nonlinear function model of Tm_Ts. All three models show strong correlation between $\mathrm{Tm}$ and $\mathrm{Ts}$. Therefore, the simulation accuracy of $\mathrm{Tm}$ is greatly affected by $\mathrm{Ts}$. Considering the correlation between air pressure, surface temperature, water vapor pressure and weighted mean temperature, the B_Tm model is related to other meteorological parameters to a certain extent and effectively improves the simulation accuracy of weighted mean temperature in China.
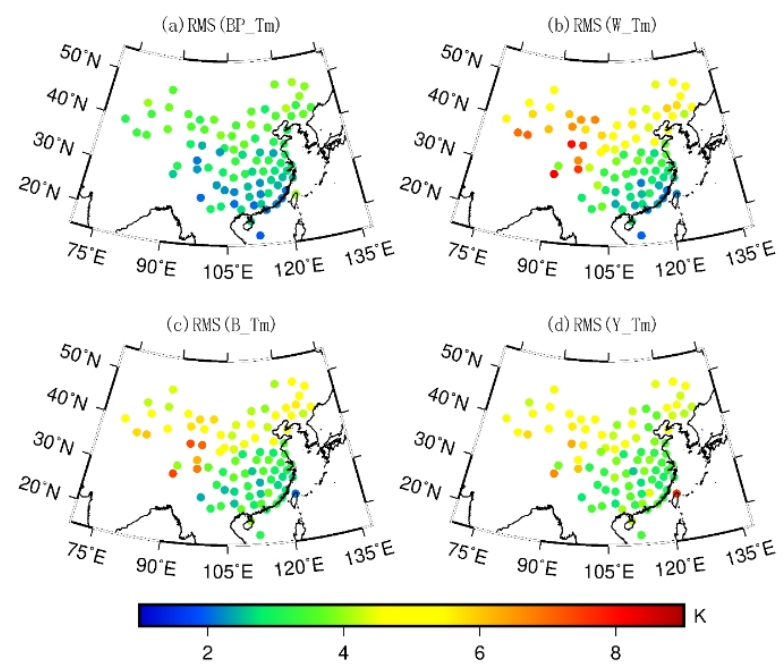

Figure 6. Distribution of RMS of different models tested using radiosonde data from 2017

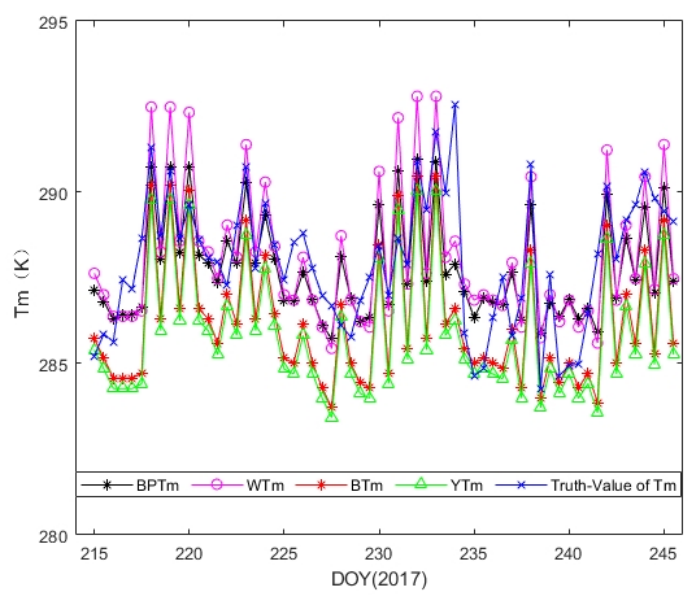

Figure 7. The analog values and true values of different models tested using radiosonde data from 2017 in Wuzhou station

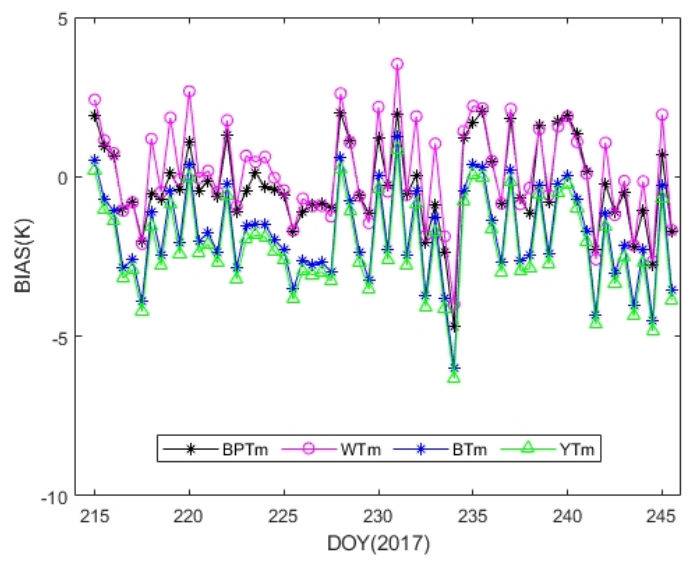

Figure 8.The bias of different models tested using radiosonde data from 2017 in Wuzhou station

\section{CONCLUSIONS}

Based on the optimized BP neural network, the initial model parameters are calculated by using the radiosonde data of 89 radiosonde stations in China in 2016. The data of 89 radiosonde stations at 0:00 and 12:00 are selected for the Chinese region in 2017. The Tm model, and a comparative analysis of the commonly used Tm linear function model and nonlinear function model, draws the following conclusions:

(1) The optimized BP_Tm model has a simple structure, fewer parameters, less data required and high operability.

(2) The root mean square error of Tm calculated by the BP_Tm model established in this paper is $3.144 \mathrm{~K}$, which improves the accuracy of B_Tm model, W_Tm 
model, and Y_Tm model by $31 \%, 34 \%$, and $22 \%$, respectively.

(3) When simulating the weighted mean temperature of a single station, the accuracy of Tm simulated by the four models is compared and analyzed. The BP_Tm model can obtain good precision and reflect better stability and reliability.

It should be noted that factors such as latitude, climate, station height difference of measurement stations, and real-time acquisition of experimental data will affect the accuracy of the weighted mean temperature model. In the next study, the model can further improve according to the influencing factors.

\section{ACKNOWLEDGEMENTS}

This work was sponsored by the National Natural Foundation o f China (41664002; 41704027); Guangxi Natural Science Foun dation of China (2018GXNSFAA294045; 2017GXNSFDA198 016; 2017GXNSFBA198139); the"Ba Gui Scholars" program of the provincial government of Guangxi; and the Guangxi Key Laboratory of Spatial Information and Geomatics (14-045-2410;16-380-25-01).

\section{REFERENCES}

Askne J, Nordius H. Estimation of tropospheric delay for microwaves from surface weather data. Radio Science, 1987, 22.

Bevis M, Businger S, Herring T A, et al. GPS meteorology: Remote sensing of atmospheric water vapor using the global positioning system. Journal of Geophysical Research Atmospheres, 1992, 97(D14):15787-15801.

Bevis M, Businger S, Chiswell S, et al. GPS Meteorology: Mapping Zenith Wet Delays onto Precipitable Water. Journal of Applied Meteorology, 1994, 33(3):379-386.

Chen P., Yao W.Q., Zhu X.J., Realization of global empirical model for mapping zenith wet delays onto precipitable water using NCEP re-analysis data. Geophysical Journal International, 2014, 198(3):1748-1757.
Davis J.L., Herring T.A., Shapiro I.I., et al. Geodesy by radio interferometry: Effects of atmospheric modeling errors on estimates of baseline length. Radio Science, 1985, 20(6).

Huang L.K., Jiang W.P., Liu L.L., et al. A new global grid model for the determination of atmospheric weighted mean temperature in GPS precipitable water vapor. Journal of Geodesy, 2018(D14).

Wang X.Y., Dai Z.Q., Cao Y.C., et al. Weighted Mean Temperature Tm Statistical Analysis in Groud-based GPS in China. Geomatics and Infor-mation Science of Wuhan University, 2011, 36(4):412-416.

Xie S.F., LI J.Y., LIU L.L., et al. Refinement of GGOS atmosphere weighted mean temperature in Xinjiang. Journal of Journal Geodesy and Geodynamics, 2017, 37(5):472-477.

$\mathrm{Yu}$ S.J., LIU L.T.. Verification and analysis on water-vapor-weighting mean temperature from $\mathrm{Tm}-\mathrm{Ts}$, relationship. Geomatics and Information Science of Wuhan University, 2009, 34(6):741-744.

Yao Y.B., Xu C., Zhang B., et al. GTm-III: a new global empirical model for mapping zenith wet delays onto precipitable water vapour. Geophysical Journal International, 2014, 197(1):202-212.

Yao Y., Zhang B., Xu C., et al. Analysis of the Global Tm-Ts, Correlation and Establishment of the Latitude-Related Linear Model. Chinese Science Bulletin, 2014, 59(19):2 340-2 347.

Yao Y.B., LIU J., Zhang B.,et al.Nonlinear relationships between the surface temperature and the weighted mean temperature. Geomatics and Information Science of Wuhan University, 2015,40(1):112-116.

Zhang H.X., Yuan Y.B., Li W, et al. GPS PPP-Derived Precipitable Water Vapor Retrieval Based on Tm/Ps from Multiple Sources of Meteorological Data Sets in China. Journal of Geoph ysical Research: Atmospheres, 2017, 122(8): 4 165-4 18 